\title{
Prévalence de la pneumonie chez les enfants de moins de 12 ans qui ont une radiographie abdominale à l'urgence
}

\author{
Valérie Homier, MD*; Colette Bellavance, $\mathrm{MD}^{* \dagger}$; Marianne Xhignesse, $\mathrm{MD}^{\dagger}$
}

\begin{abstract}
RÉSUMÉ
Objectif : La pneumonie est une cause bien connue de douleur abdominale aiguë chez les enfants. L'utilité de la radiographie pulmonaire dans ce contexte est toutefois controversée. Nous avons cherché à déterminer la prévalence de la pneumonie chez les enfants de moins de 12 ans qui souffraient de douleur abdominale et ont eu une radiographie abdominale à l'urgence. Nous voulions aussi décrire les signes et les symptômes des enfants chez lesquels on a diagnostiqué une pneumonie dans ce contexte.

Méthodes : Nous avons procédé à une analyse rétrospective des données électroniques tirées des visites effectuées à l'urgence d'un centre de soins tertiaires par des enfants de 12 ans et moins examinés entre le 1er juin 2001 et le 30 juin 2003 et qui ont eu une radiographie abdominale et pulmonaire au cours de la même visite, ou une radiographie abdominale au cours d'une première visite et une radiographie pulmonaire dans les 10 jours suivant la visite initiale.

Résultats : Sur les 1584 visites étudiées, on a repéré 30 cas de pneumonie, ce qui représente une prévalence de 1,89\% (intervalle de confiance à $95 \%, 1,22 \%-1,56 \%$ ). Si on avait limité la radiographie pulmonaire aux enfants qui avaient de la fière, toussaient et présentaient des symptômes d'infection des voies respiratoires supérieures (IVRS), on aurait raté le diagnostic de pneumonie dans seulement 2 cas sur $1584(0,13 \%)$.

Conclusion : Les enfants de 12 ans et moins qui se présentent à l'urgence avec une douleur abdominale aiguë et pour lesquels on demande une radiographie abdominale n'ont besoin que d'une radiographie pulmonaire s'ils toussent, ont de la fière ou présentent d'autres symptômes d'IVRS.
\end{abstract}

Mots clés : douleur abdominale, enfants, urgence, pneumonie, radiographie pulmonaire

\section{Introduction}

La pneumonie est une cause bien connue de douleur abdominale chez l'enfant. ${ }^{1-8}$ Une recension des textes indique qu'on ne s'entend toujours pas sur la nécessité de soumet- tre à une radiographie pulmonaire les enfants qui se présentent à l'urgence en se plaignant d'une douleur abdominale. Des auteurs sont en faveur d'une telle pratique, ${ }^{9-12}$ tandis que d'autres ne recommandent pas d'effectuer d'emblée une radiographie des poumons. ${ }^{13-17}$ \footnotetext{
Sherbrooke (Qué.).

Reçu le 5 février 2006; révisions reçues le 23 août 2006; accepté le 15 octobre 2006.

Cet article a été soumis à l'examen des pairs.

Can J Emerg Med 2007;9(5)
}

De *la Faculté de médecine, Université de Sherbrooke, Sherbrooke (Qué.) et ¥du Centre hospitalier universitaire de Sherbrooke 
En novembre 2000, le Centre hospitalier universitaire de Sherbrooke (CHUS), Hôpital Fleurimont, a adopté une politique sur les enfants de 12 ans ou moins qui se présentent à l'urgence avec une douleur abdominale. Cette politique imposait une radiographie pulmonaire conjuguée à toute radiographie abdominale afin d'exclure la pneumonie chez ces enfants.

Cette étude visait à déterminer la prévalence de la pneumonie chez les enfants qui se présentent à l'urgence en se plaignant d'une douleur abdominale et qui se soumettent à une radiographie abdominale, ainsi qu'à décrire les signes et les symptômes que présentent les sujets dont la radiographie pulmonaire donne un résultat positif.

\section{Méthodes}

On a procédé à une analyse rétrospective à partir de la base de données informatisée de l'hôpital (Nucleus). L'étude visait les enfants de 0 à 12 ans qui se sont présentés à l'urgence, entre le $1^{\text {er }}$ juin 2001 et le 30 juin 2003, en se plaignant principalement d'une douleur abdominale et qui ont eu une radiographie pulmonaire et abdominale au moment de leur visite à l'urgence (groupe 1) ou une radiographie abdominale seulement au cours de leur visite à l'urgence et une radiographie pulmonaire dans les 10 jours suivant la première visite (groupe 2).

Le chercheur principal a lu le rapport final du radiologiste pour déterminer le nombre de cas de pneumonie. Il n'y a pas eu d'évaluation à l'aveugle par un deuxième radiologiste au moment de la collecte des données. On a réuni les renseignements suivants sur chaque cas de pneumonie : âge du patient, type de radiographie qui a déterminé le diagnostic de pneumonie (abdominale ou pulmonaire), site de la pneumonie, numérations leucocytaire et granulocytaire du patient, date de la visite et numéro de dossier médical chiffré.

On a entré les données dans des fichiers Excel (Microsoft) distincts. Le premier fichier (groupe 1) contenait les patients qui ont subi une radiographie pulmonaire et abdominale au cours de la visite initiale $(n=1603)$. Le deuxième (groupe 2) contenait ceux qui ont eu une radiographie pulmonaire au cours des 10 jours qui ont suivi la visite à l'urgence ( $n=31)$.

Dans les cas où l'interprétation du radiologiste n'était pas un diagnostic (p. ex., présence d'une opacité rétrocardiaque non spécifique pouvant représenter une atélectasie ou un début de pneumonie), on a étudié le dossier pour confirmer ou exclure la possibilité d'une pneumonie. Cette approche a permis d'extraire des renseignements cliniques comme la présence et la localisation de douleur abdomi- nale, les signes vitaux au moment du triage, les antécédents de fièvre, la présence d'une toux et de symptômes d'infection des voies respiratoires supérieures (IVRS) (c.-à-d. rhinorrhée, congestion nasale, mal de gorge et otalgie). Au total, on a étudié 60 dossiers. Les chercheurs ont discuté de leurs constatations et ont dégagé un consensus.

On a déterminé qu'il y avait fièvre au moment du triage lorsque la température dépassait $38,5{ }^{\circ} \mathrm{C}$ prise par voie rectale ou $38{ }^{\circ} \mathrm{C}$ prise par voie orale. Le CHUS (Hôpital Fleurimont) a fourni les numérations leucocytaire et granulocytaire normales en fonction de l'âge en juillet 2003 pour permettre de définir les valeurs anormales. On a défini la tachypnée spécifique à l'âge en fonction de la plage de référence fournie dans le Guide de l'examen clinique. ${ }^{18}$

On n'a effectué aucune analyse statistique dans le cadre de cette étude.

\section{Considérations éthiques}

Le directeur des services professionnels du CHUS a approuvé cette étude dans le contexte d'un projet d'amélioration de la qualité.

\section{Résultats}

Dans le groupe 1, nous avons identifié 1401 enfants de 0 à 12 ans qui ont effectué 1603 visites à l'urgence entre le $1^{\text {er }}$ juin 2001 et le 30 juin 2003 et ont eu une radiographie abdominale et pulmonaire au cours de la même visite. Nous avons exclu 19 visites parce que les rapports de radiographie manquaient. Nous avons exclu 10 autres cas sans étudier le dossier parce qu'ils représentaient une pneumonie nosocomiale ou que les patients étaient déjà traités pour une pneumonie lorsqu'ils se sont présentés. Nous avons exclu trois autres cas après étude des dossiers : deux parce que les patients étaient déjà traités pour une pneumonie et l'autre parce qu'il n'était aucunement question de douleurs abdominales dans les notes du médecin.

Chez les sujets du groupe 2, 31 enfants ont eu une radiographie abdominale au moment de leur visite à l'urgence et ensuite une radiographie pulmonaire dans les 10 jours suivant la première visite. Nous avons exclu deux dossiers de ce groupe parce que les rapports de radiographie manquaient. Nous avons repéré un seul cas de pneumonie dans les 29 dossiers restants. Nous l'avons exclu parce qu'il s'agissait d'un transfert, en provenance d'un autre établissement, d'un patient qui avait eu des radiographies à l'hôpital d'origine. Le Tableau 1 présente un aperçu des méthodes d'étude. Toutes ces exclusions reposaient sur des critères établis dans le contexte du protocole d'étude.

Sur les 1584 visites que nous avons analysées dans le 
groupe 1 , nous avons repéré 30 cas de pneumonie, ce qui donne une prévalence de $1,89 \%$ (intervalle de confiance [IC] à $95 \%, 1,22 \%-1,56 \%)$. Le médecin de l'urgence a raté au début quatre de ces cas de pneumonie diagnostiqués par radiographie. Le Tableau 2 présente les caractéristiques des patients, ainsi que les signes et symptômes des 30 enfants atteints de pneumonie.

Nous avons résumé la prévalence des symptômes d'IVRS (c.-à-d. toux, fièvre, tachypnée, faible saturation en oxygène et numérations leucocytaire et granulocytaire) pour les 30 enfants du groupe 1 qui avaient une pneumonie (Tableau 3). La fièvre était le signe que l'on associait le plus à la pneumonie et elle était présente dans $83 \%$ des cas.

Nous avons considéré comme négatives les données manquantes à l'égard de chaque signe et symptôme et nous avons calculé la prévalence en divisant le nombre de cas présentant ces signes et symptômes par 30 et en convertissant ensuite cette valeur en pourcentage. Nous avons adopté cette stratégie afin de minimiser l'effet de ces indicateurs.

Les caractéristiques cliniques notées au cours de cette étude et indiquées par ordre de prévalence décroissante sont la fièvre, les symptômes d'IVRS et la toux. Si on avait limité la radiographie pulmonaire, en sus d'une radiogra-

Tableau 1. Prévalence de la pneumonie chez les enfants de moins de 12 ans qui ont eu une radiographie abdominale à l'urgence*

\begin{tabular}{|c|c|c|}
\hline \multirow[b]{2}{*}{ Variable } & \multicolumn{2}{|c|}{ Nbre de patientst } \\
\hline & Groupe 1 & Groupe 2 \\
\hline Radiographie, nbre de cas $\ddagger$ & 1603 & 31 \\
\hline Exclusions§ & 19 & 2 \\
\hline Total & 1584 & 29 \\
\hline \multicolumn{3}{|l|}{$\begin{array}{l}\text { Interprétation du } \\
\text { radiologiste }\end{array}$} \\
\hline Pneumonie & 43 & 1 \\
\hline $\begin{array}{l}\text { Exclusions sans étude } \\
\text { du dossierףl }\end{array}$ & 10 & 0 \\
\hline Total & 33 & 1 \\
\hline $\begin{array}{l}\text { Exclusions } \\
\text { supplémentaires après } \\
\text { étude du dossier }\end{array}$ & $3 * *$ & $1 †+$ \\
\hline Total & 30 & 0 \\
\hline \multicolumn{3}{|c|}{ 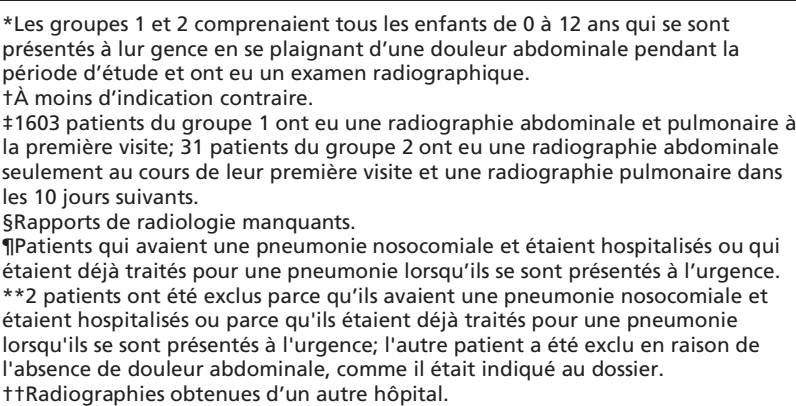 } \\
\hline
\end{tabular}

phie abdominale, aux enfants qui avaient de la fièvre ou une toux, ou des symptômes d'IVRS, on aurait raté seulement 2 cas sur 1584 patients $(0,13 \%)$ chez lesquels on a diagnostiqué finalement une pneumonie.

\section{Discussion}

La prévalence de la pneumonie signalée dans le contexte de cette étude s'établit à 1,89\% (IC à $95 \%, 1,22-1,56 \%$ ) et correspond à celle que l'on retrouve dans les publications. Sims et Alexander ${ }^{19}$ ont signalé une prévalence de $2 \%$; Jones $^{20}$ a indiqué 2,2 \%; et Ravichandran et Burge, ${ }^{15} 1,6 \%$. Étant donné la faible prévalence de la pneumonie chez les enfants qui se plaignent de douleurs abdominales, nous sommes d'avis, tout comme Hayes, ${ }^{13}$ Spencer ${ }^{14}$ et Ravichandran et Burge,,$^{15}$ qu'il est inutile de soumettre à une radiographie pulmonaire de routine les enfants qui se présentent en se plaignant d'une douleur abdominale isolée.

Les auteurs de trois études moins récentes recommandent une radiographie pulmonaire pour tous les enfants qui se plaignent d'une douleur abdominale. ${ }^{9-11}$ Deux de ces études signalent une prévalence plus élevée de pneumonie dans leur contexte $(4,8 \%$ et $4,2 \%) .^{10,11}$ La troisième étude ne mentionne pas de prévalence ni l'âge des enfants. ${ }^{9}$

Ni Spencer, ${ }^{14}$ ni Vendargon et ses collaborateurs16 n'appuient le concept de la radiographie pulmonaire chez les enfants qui se plaignent d'une douleur abdominale aiguë, même s'ils fondent leur opinion sur des rapports de cas seulement. Ravichandran et Burge ${ }^{15}$ sont du même avis, mais ils n'ont pas établi d'étalon-or pour le diagnostic dans le contexte de leur travail. Notre étude réfute la nécessité des radiographies pulmonaires de routine, ce qui renforce la position adoptée dans les publications et présente une perspective plus récente sur la question.

Il convient de discuter de certaines limites de l'étude. Comme dans toute étude rétrospective, les données aux dossiers seront incomplètes. Il se peut que dans certains cas, la politique n'ait pas été bien appliquée et que quelques enfants se plaignant de douleurs abdominales aient eu une radiographie abdominale sans radiographie pulmonaire. L'étude a de plus été réalisée dans un seul centre, ce qui limite la validité externe. Une étude prospective réalisée dans de multiples hôpitaux permettrait de confirmer ces résultats.

\section{Conclusion}

Dans le contexte de cette étude, nous avons établi à 1,89\% (IC à $95 \%, 1,22-1,56 \%$ ) la prévalence de la pneumonie chez les enfants âgés de 0 à 12 ans qui se sont présentés à 
Tableau 2. Caractéristiques, signes et symptômes chez 30 cas de pneumonie du groupe 1 .

\begin{tabular}{|c|c|c|c|}
\hline $\begin{array}{l}\text { Caractéristiques, signes ou } \\
\text { symptômes }\end{array}$ & $\begin{array}{l}\text { Nbre de } \\
\text { patients* }\end{array}$ & Caractéristiques, signes ou symptômes & $\begin{array}{l}\text { Nbre de } \\
\text { patients* }\end{array}$ \\
\hline Sexe & & Toux & \\
\hline Mâle & 20 & Présence & 20 \\
\hline Femelle & 10 & Absence & 1 \\
\hline Âge & & Information non documentée & 9 \\
\hline Moyenne & 4,7 ans & Symptômes d'IFRS (rhinorrhée, congestion, mal de gorge c & talgie) \\
\hline Intervalle & $9 m-12$ ans & Présence de symptômes & 23 \\
\hline Diagnostique de pneumonie & & Absence de symptômes & 2 \\
\hline $\begin{array}{l}\text { Visible seulement sur la } \\
\text { radiographie pulmonaire }\end{array}$ & 28 & Information au sujet des symptômes non documentée & 5 \\
\hline $\begin{array}{l}\text { Visible aussi sur la radiographie } \\
\text { abdominale }\end{array}$ & 2 & $\begin{array}{l}\text { Fièvre }\left(>38,5^{\circ} \mathrm{C} \text { rectale ou }>38^{\circ} \mathrm{C} \text { orale) selon les }\right. \\
\text { antécédents ou documentée au triage }\end{array}$ & \\
\hline Atteinte des lobes & & Présence & 25 \\
\hline Lobe inférieur gauche & 13 & Absence & 5 \\
\hline Lobe moyen gauche & 4 & Saturation en oxygène & \\
\hline Lobe inférieur droit & 4 & $<95 \%$ & 6 \\
\hline Autre & 9 & $>95 \%$ & 17 \\
\hline Douleur abdominale & & Non documentée & 7 \\
\hline Documentée dans le dossier & 29 & Fréquence respiratoireł & \\
\hline $\begin{array}{l}\text { Pas de douleur abdominale } \\
\text { documentéet }\end{array}$ & 1 & Élevée par rapport à l'âge & 5 \\
\hline Site de la douleur abdominale & & Normale par rapport à l'âge & 11 \\
\hline Diffuse & 7 & Non documentée & 14 \\
\hline Périombilicale & 5 & Compte de globules blancs et de granulocytes§ & \\
\hline Quadrant inférieur droit & 4 & Compte anormal de globules blancs et de granulocytes & 6 \\
\hline Autre & 6 & $\begin{array}{l}\text { Compte normal de globules blancs et } \\
\text { anormal de granulocytes }\end{array}$ & 7 \\
\hline Site non documenté & 8 & Compte normal de globules blancs et de granulocytes & 8 \\
\hline & & Données non disponibles & 9 \\
\hline \multicolumn{4}{|c|}{$\begin{array}{l}\text { IVRS = infection des voies respiratoires supérieures. } \\
\text { *A moins d'indication contraire. } \\
\text { tPatient âgé d'un an. } \\
\text { tValeurs normales tirées du Guide de lexamen clinique }{ }^{15} \text {. } \\
\text { §Valeurs normales du compte de globules blancs et de granulocytes du laboratoire d'hématologie du Centre hospitalier universitaire de Sherbrooke (Hôpital } \\
\text { Fleurimont) en juillet } 2003 \text {. }\end{array}$} \\
\hline
\end{tabular}

\begin{tabular}{|c|c|c|c|}
\hline Signe ou symptôme & $\begin{array}{l}\text { Données } \\
\text { disponibles du } \\
\text { group } 1 \\
(n=30)\end{array}$ & $\begin{array}{l}\text { Nbre d'enfants } \\
\text { présentant le } \\
\text { signe ou le } \\
\text { symptôme }\end{array}$ & $\begin{array}{l}\text { Prévalence } \\
\text { du signe } \\
\text { ou du } \\
\text { symptôme }\end{array}$ \\
\hline Toux & 21 & 20 & $67 \%$ \\
\hline IVRS & 25 & 23 & $77 \%$ \\
\hline Fièvre & 30 & 25 & $83 \%$ \\
\hline Tachypnée & 16 & 5 & $17 \%$ \\
\hline Baisse de la saturation & 23 & 6 & $20 \%$ \\
\hline $\begin{array}{l}\text { Compte élevé de } \\
\text { globules blancs et(ou) } \\
\text { de granulocytes }\end{array}$ & 21 & 12 & $40 \%$ \\
\hline IVRS ou toux & 26 & 26 & $87 \%$ \\
\hline IVRS ou fièvre & 30 & 27 & $90 \%$ \\
\hline Toux ou fièvre & 30 & 28 & $93 \%$ \\
\hline IVRS ou toux ou fièvre & 30 & 28 & $93 \%$ \\
\hline
\end{tabular}


l'urgence d'un centre de soins tertiaires, entre le $1^{\text {er }}$ juin 2001 et le 30 juin 2003, et ont eu une radiographie abdominale parce qu'ils se plaignaient principalement d'une douleur abdominale. La fièvre est le signe que l'on a associé le plus étroitement à la pneumonie et était présente dans $83 \%$ des cas. Il n'est pas nécessaire de soumettre systématiquement les enfants de cet âge qui se présentent à l'urgence en se plaignant d'une douleur abdominale isolée à une radiographie pulmonaire en plus de la radiographie abdominale. Il se peut plutôt que la radiographie pulmonaire ne soit nécessaire que si l'enfant présente aussi des signes de toux, de fièvre ou d'IVRS.

Remerciements : nous tenons à remercier Hassan Diab, $\mathrm{PhD}$, administrateur principal, Système d'aide à la décision, Centre de recherche clinique du CHUS, pour son importante contribution à la réalisation de ce projet de recherche.

Intérêts concurrents : aucun déclaré.

\section{Références}

1. Purcell TB. Nonsurgical and extraperitoneal causes of abdominal pain. Emerg Med Clin North Am 1989;7:721-40.

2. Kirschner BS. The gastrointestinal tract. Dans : Nelson essentials of pediatrics. $4^{\mathrm{e}}$ éd. Behrman RE, Kliegman RM (sous la direction de). Philadelphie (PA) : W.B. Saunders Company; 2002. p. 469-71.

3. Black DD. The respiratory system. Dans : Nelson essentials of pediatrics. $4^{\mathrm{e}}$ éd. Behrman RE, Kliegman RM (sous la direction de). Philadelphie (PA) : W.B. Saunders Company, 2002. p. 541-4.

4. Prober CG. Pneumonia. Dans : Nelson textbook of pediatrics. $16^{\mathrm{e}}$ éd. Behrman RE, Kliegman RM, Jenson HB (sous la direction de). Philadelphie (PA) : W.B. Saunders Company, 2000. p. 761-5.

5. Ulshen M. Major signs and symptoms of digestive tract disorders. Dans : Nelson textbook of pediatrics. $16^{\mathrm{e}}$ éd. Behrman RE, Kliegman RM, Jenson HB (sous la direction de). Philadelphie (PA) : W.B. Saunders Company; 2000. p. 1102-6.
6. Lillis KA. Gastrointestinal disorders. Dans : Barkin RM (sous la direction de). Pediatric emergency medicine: concepts and clinical practice. $2^{\mathrm{e}}$ éd. St. Louis (MO) : Mosby-Year Book Inc.; 1997. p. 797-804.

7. Letourneau MA, Schuh S, Gausche M. Respiratory disorders. Dans : Barkin RM (sous la direction de). Pediatric emergency medicine: concepts and clinical practice. $2^{\mathrm{e}}$ éd. St. Louis (MO) : Mosby-Year Book, Inc; 1997. p. 1102-8.

8. Ruddy RM. Pain-abdomen. Dans : Textbook of pediatric emergency medicine. $4^{\mathrm{e}}$ éd. Feisher GR, Ludwig S (sous la direction de). Philadelphie (PA) : Lippincott Williams \& Wilkins; 2000. p. 421-8.

9. Freimanis AK, Nelson SW. The chest roentgenogram in the diagnosis of acute abdominal disease. Radiol Clin North Am 1964;2:3-20

10. Jona JZ, Belin RP. Basilar pneumonia simulating acute appendicitis in children. Arch Surg 1976;111:552-3.

11. Jona JZ, Selke AC, Belin RP. Radiologic aids in the diagnosis of appendicitis in children. South Med J 1975;68:1373-6.

12. Swischuk LE. Emergency imaging of the acutely ill or injured child. $3^{\mathrm{e}}$ éd. Baltimore (MD) : Williams \& Wilkins; 1994. p. 222-3.

13. Hayes R. Abdominal pain: general imaging strategies. Eur Radiol 2004;14:L123-37.

14. Spencer PAS. Pneumonia, diagnosed on the abdominal radiograph, as a cause for acute abdomen in children. Br J Radiol 1990;63:306-8.

15. Ravichandran D, Burge DM. Pneumonia presenting with acute abdominal pain in children. Br J Surg 1996;83:1707-8.

16. Vendargon S, Wong PS, Tan KK. Pneumonia presenting as acute abdomen in children: a report of three cases. Med J Malaysia 2000;55:520-3.

17. Cope's early diagnosis of the acute abdomen. $20^{\mathrm{e}}$ éd. revue par William Silen. New York (NY) : Oxford University Press; 2000. p. 58, 273-5.

18. Bates B. Guide de l'examen clinique, $4^{\mathrm{e}}$ édition. Reuil-Malmaison : Arnette, 2001. p. 739.

19. Sims DG, Alexander FW. Acute abdominal pain in childhood. BMJ 1976;2:880-1.

20. Jones PF. Active observation in management of acute abdominal pain in childhood. BMJ 1976;2:551-3.

Correspondance : $\mathrm{D}^{\mathrm{r}}$ Valérie Homier, Hôpital Royal Victoria, 687, avenu des Pins Ouest, bureau A4.62, Montréal (Qué.) H3A 1A1; valerie.homier@mail.mcgill.ca 